Bull. Austral. Math. Soc.

VOL. 55 (1997) [469-476]

\title{
COVERING GROUPS WITH SUBGROUPS
}

\author{
R.A. Bryce, V. Fedri and L. Serena
}

A group is covered by a collection of subgroups if it is the union of the collection. The intersection of an irredundant cover of $n$ subgroups is known to have index bounded by a function of $n$, though in general the precise bound is not known. Here we confirm a claim of Tompkinson that the correct bound is 16 when $n$ is 5 . The proof depends on determining all the 'minimal' groups with an irredundant cover of five maximal subgroups.

\section{INTRODUCTION}

A covering or cover of a group $G$ is a collection of subgroups of $G$ whose union is $G$. We use the term $n$-cover for a cover with $n$ members. The cover is irredundant if no proper sub-collection is also a cover. Neumann [5] obtained a uniform bound for the index of the intersection of an irredundant $n$-cover; see Tompkinson [7] for an improved bound. We shall write $f(n)$ for the largest index $|G: D|$ over all groups $G$ with an irredundant $n$-cover with intersection $D$. An immediate consequence is that such a group $G$ has a permutation representation of degree at most $f(n)$, with kernel $\operatorname{core}_{G}(D)$. In particular $G / \operatorname{core}_{G}(D)$ is a finite group with an irredundant $n$-cover whose intersection is core-free.

The groups with an irredundant core-free intersection covering are known precisely when $n=3$ (Scorza [6]) and when $n=4$ (Greco [4, p.58]): see Propositions 2.3 and 2.4 below. Partial results are known for $n=5$ : Greco [3] lists all groups with an irredundant 5-cover in which all pairwise intersections are the same; and Tompkinson [7] claims that $f(5)=16$.

The aim of the present article is to fill in some of the missing detail when $n=5$. We are concerned with irredundant, core-free intersection 5-covers in which all five subgroups of the cover are maximal. A cover in which all subgroups are maximal we shall call maximal.

THEOREM 1.1. Let $G$ be a group with a maximal irredundant cover of five subgroups with core-free intersection $D$. Then either

(a) $D=1$ and $G$ is elementary Abelian of order 16; or

(b) $D=1$ and $G \cong \mathrm{Alt}_{4}$; or

(c) $|D|=3,|G|=48$ and $G$ embeds in $\mathrm{Alt}_{4} \times \mathrm{Alt}_{4}$.

Received 24 June 1966

Copyright Clearance Centre, Inc. Serial-fee code: $0004-9729 / 97 \quad \$ A 2.00+0.00$ 
THEOREM 1.2. $f(5)=16$.

\section{Preliminary Results}

The following results will be needed below. Where no proof is given it is either very easy or a reference is given.

LEMMA 2.1. Let $\left\{A_{i}: 1 \leqslant i \leqslant m\right\}$ be a (maximal) irredundant covering of a group $G$ with intersection $D$. If $N$ is a normal subgroup of $G$ contained in $D$ then $\left\{A_{i} / N: 1 \leqslant i \leqslant m\right\}$ is a (maximal) irredundant cover of $G / N$.

Lemma 2.2. (See [1, Lemma 2.2]) Let $\mathcal{A}=\left\{A_{i}: 1 \leqslant i \leqslant m\right\}$ be an irredundant covering of a group $G$ whose intersection is $D$.

(a) If $p$ is a prime, $x$ a $p$-element of $G$ and $\left|\left\{i: x \in A_{i}\right\}\right|=n$ then either $x \in D$ or $p \leqslant m-n$.

(b) $\bigcap_{j \neq i} A_{j}=D(1 \leqslant i \leqslant m)$.

(c) If $\bigcap_{i \in S} A_{i}=D$ whenever $|S|=n$ then $\left|\bigcap_{i \in T} A_{i}: D\right| \leqslant m-n+1$ whenever $|T|=n-1$.

(d) If $\mathcal{A}$ is maximal and $U$ is an Abelian minimal normal subgroup of $G$ then, if $\left|\left\{i: U \leqslant A_{i}\right\}\right|=n$, either $U \subseteq D$, or $|U| \leqslant m-n$.

Proposition 2.3. (Scorza [6]) Let $\left\{A_{i}: 1 \leqslant i \leqslant 3\right\}$ be an irredundant cover with core-free intersection $D$ of a group $G$. Then $D=1$ and $G \cong C_{2} \times C_{2}$.

Proposition 2.4. (Greco [4]) Let $\left\{A_{i}: 1 \leqslant i \leqslant 4\right\}$ be an irredundant cover with core-free intersection $D$ of a group $G$. If the cover is maximal then either

(a) $D=1$ and $G \cong \mathrm{Sym}_{3}$ or $G \cong C_{3} \times C_{3}$; or

(b) $|D|=2,|G|=18$ and $G$ embeds into $\mathrm{Sym}_{3} \times \mathrm{Sym}_{3}$.

If the cover is not maximal then either

(c) $D=1$ and $G \cong D_{8}$, or $G \cong C_{4} \times C_{2}$, or $G \cong C_{2} \times C_{2} \times C_{2}$; or

(d) $|D|=2$ and $G \cong D_{8} \times C_{2}$.

LEMMA 2.5. Let $G$ be a group with a maximal irredundant 5-cover with corefree intersection $D$.

(a) $G$ is a 2-group if and only if $D=1$ and $G$ is elementary of order 16 .

(b) $G$ is not a 3-group.

ProOF: Let $G=M_{1} \cup M_{2} \cup M_{3} \cup M_{4} \cup M_{5}$ be a maximal irredundant cover for a p-group $G$, with core-free intersection $D$. Now $\Phi(G) \subseteq D$ so $D \unlhd G$, therefore $D=1$, and $G$ is elementary Abelian. By Lemma 2.2(b), (c), $\left|M_{i} \cap M_{j} \cap M_{k}\right| \leqslant 2$ whenever $i, j, k$ are distinct. When $p=2$, therefore, $|G| \leqslant 16$. Also $|G| \geqslant 8$ since otherwise $G$ 
does not have five maximal subgroups. However $|G|=8$ is impossible. For, if $|G|=8$ and $\left|M_{1} \cap M_{2} \cap M_{3}\right|=2$ then $G=M_{1} \cup M_{2} \cup M_{3}$, contradicting the irredundance of the cover; and if $M_{1} \cap M_{2} \cap M_{3}=1$ then $\left|M_{1} \cup M_{2} \cup M_{3}\right|=7$, so $G$ is covered by four of the $M_{i}$, again a contradiction. Conversely if $\langle a, b, c, d\rangle$ is elementary of order 16 , then $\langle a, b, c\rangle,\langle a, b, d\rangle,\langle a, c, d\rangle,\langle b, c, d\rangle,\langle a b, b c, c d\rangle$ provide a maximal irredundant core-free intersection cover.

When $p=3$ we conclude that $M_{i} \cap M_{j} \cap M_{k}=1$ for all distinct $i, j, k .|G|>9$ since an elementary Abelian group of order 9 has only four maximal subgroups; in particular, no pairwise intersection is trivial. Hence $\left|M_{i} \cap M_{j}\right|=3(i \neq j)$. By the inclusion-exclusion principle $|G|=5.9-10.3+10.1-5.1+1=21$, which is not a power of 3 , a contradiction.

Lemma 2.6. Let $F$ be finite field with $q$ elements. Suppose that

$$
F^{2}=S_{1} \cup S_{2} \cup \ldots \cup S_{m}
$$

where $S_{i}$ is a translate of a one dimensional subspace $U_{i}(1 \leqslant i \leqslant m)$. Then $m \geqslant q$ and

(a) if $m=q, U_{1}=U_{i}(1 \leqslant i \leqslant q)$;

(b) if $m=q+1$ and the union (2.1) is irredundant, then the subspaces $U_{i}$ are distinct and, for some $r \in F, S_{i}=U_{i}+r(1 \leqslant i \leqslant q+1)$;

and

(c) if $m=q+2$ and the union (2.1) is irredundant then the subspaces $U_{i}$ $(1 \leqslant i \leqslant q+2)$ do not cover $F^{2}$.

Proof: Firstly note that $m q \geqslant q^{2}$ so $m \geqslant q$. Now observe that $F^{2}$ can be thought of as an affine plane in which the lines are the translates of one-dimensional vector subspaces. The result then has an easy, and presumably well known, geometrical proof. We give a sketch.

(a) In this case the space is covered by the $q$ lines $S_{i}$, each containing exactly $q$ points. Hence these lines are parallel and one of them passes through the origin.

(b) We are to prove that $q+1$ lines have a common point if their union is irredundant and equal to $F^{2}$. There are at most $q$ mutually parallel lines, so $S_{1}$ and $S_{2}$ say, meet at a point $P$. Let $A=S_{1} \cup S_{2}$. Every line $S_{i}(3 \leqslant i \leqslant q+1)$ meets $A$ in at least one point. Since $\left|F^{2} \backslash A\right|=q^{2}-(2 q-1)=(q-1)^{2}$ no line $S_{i}(3 \leqslant i \leqslant q+1)$ meets $A$ in more than one point. If $q=2$ then $S_{3}$ is incident with $P$ since neither $S_{1}$ nor $S_{2}$ is redundant. Hence we may suppose that $q>2$. Now no two $S_{i}(3 \leqslant i \leqslant q+1)$ meet outside $A$. Suppose $P \in S_{i}$ but $P \notin S_{j}$ for some $i, j$ satisfying $3 \leqslant i, j \leqslant q+1$. Then $S_{j}$ is parallel to just one of $S_{1}, S_{2}$, say to $S_{1}$, and also parallel to just one of $S_{2}$, $S_{i}$ therefore to $S_{i}$, a contradiction since $S_{1}$ and $S_{i}$ are not parallel. That is, if three of 
the lines $S_{i}(1 \leqslant i \leqslant q+1)$ pass through $P$ then all do, and we are done. Suppose that none of $S_{i}(3 \leqslant i \leqslant q+1)$ is incident with $P$. Then all $S_{j}(3 \leqslant j \leqslant d)$ are parallel to $S_{1}$ and all $S_{k}(d+1 \leqslant k \leqslant q+1)$ are parallel to $S_{2}$, for some $d$ satisfying $3 \leqslant d<q+1$, or else the union (2.1) is redundant. It follows that $\left|S_{i} \cap S_{j}\right|=1$ if $i \in\{1,3, \ldots, d\}$ and $j \in\{2, d+1, \ldots, q+1\}$, and is zero otherwise; in particular all three-fold intersections are empty. Hence, counting points, $q^{2}=q(q+1)-(d-1)(q-d+2)$ whence $q=(d-1)(q-d+2)$. However, both right-side factors are greater than 1 , and hence have a prime common factor which therefore divides both $q$ and $q+1$, a contradiction.

(c) In this case $q>2$. Two of the lines, say $S_{1}$ and $S_{2}$, are parallel. It is enough to show that there is another pair of parallels. If there is not, all the lines $S_{i}(3 \leqslant i \leqslant q+2)$ are incident in pairs, and each is incident with each of $S_{1}$ and $S_{2}$. Since the complement of $S_{1} \cup S_{2}$ has cardinality $q^{2}-2 q=q(q-2)$, it follows that $S_{i} \cap S_{j} \subseteq S_{1} \cup S_{2} \quad(3 \leqslant i<j \leqslant q+2)$. If all these intersections are the same, say lying in $S_{1}$, then counting shows that $S_{2}$ is redundant. Hence $S_{1} \cap S_{h} \neq S_{1} \cap S_{k}$ for some $h, k \in\{3, \ldots, q+2\}$. Then $S_{h} \cap S_{k}$ is incident with $S_{2}$, and there is some $S_{t}(3 \leqslant t \leqslant q+2)$ for which $S_{2} \cap S_{t} \neq S_{2} \cap S_{h}$. But then one of $S_{h} \cap S_{t}$ or $S_{k} \cap S_{t}$ is not incident with $S_{1}$, a contradiction.

Lemma 2.7. Let $G$ be a group with the following structure: $O_{3}(G)$ is elementary Abelian of index 2 in $G$, and $G$ has trivial centre. There does not exist a maximal irredundant 5-cover of $G$.

Proof: Let us suppose that the result is false, and that $G$ is a minimal counterexample. Note that $|G|>6$ since $\mathrm{Sym}_{3}$ is not a counterexample.

Let

$$
G=M_{1} \cup M_{2} \cup M_{3} \cup M_{4} \cup M_{5}
$$

be a maximal irredundant cover of $G$ with core-free intersection $D$. Then $\left|M_{i}\right|>$ $2(1 \leqslant i \leqslant 5)$. Therefore either

(a) for some $i, M_{i}=V:=O_{3}(G)$ and $\left|G: M_{j}\right|=3(j \neq i)$; or

(b) $\left|G: M_{j}\right|=3$ for all $j$.

Now $D \cap V=1$ by Lemma 2.1 since $D \cap V \unlhd G$. Let $a$ be an involution of $G$. Since $\langle a\rangle$ is a Sylow 2-subgroup of $G$ every 2-element of $G$ is conjugate to $a$. Define

$$
S_{i}:=\left\{x \in V: a^{x} \in M_{i}\right\}, \quad 1 \leqslant i \leqslant 5 .
$$

Either $S_{i}=\emptyset$ or $S_{i}$ is a coset of $X_{i}:=V \cap M_{i}$ in $V$, and there is at most one of the first type. For all $x \in V$, there is an $i$ for which $a^{x} \in M_{i}$ so

$$
V=S_{1} \cup S_{2} \cup S_{3} \cup S_{4} \cup S_{5} .
$$


From Lemma 2.2(c) the intersection of every triple of the subgroups $X_{i}(1 \leqslant i \leqslant 5)$ is trivial. In the case (a) suppose that $M_{5}=V$, so that the pairwise intersections $X_{i} \cap X_{j}(1 \leqslant i<j \leqslant 4)$ are all trivial. In particular $|V|=9$. Also $S_{5}=\emptyset$ and

$$
V=S_{1} \cup S_{2} \cup S_{3} \cup S_{4}
$$

In this union all the $S_{i}$ are essential since if, say, $S_{1}$ were omissible, then $M_{1}$ would be omissible in (2.2). However Lemma 2.6 now shows that the subgroups $X_{i}(1 \leqslant i \leqslant 4)$ are distinct. They therefore cover $V$ making $M_{5}$ redundant, a contradiction. This shows that case (a) does not arise.

In case (b) we have $V=X_{1} \cup X_{2} \cup X_{3} \cup X_{4} \cup X_{5}$. From Lemma 2.5 this union is redundant; and from Proposition 2.3 just one term, say $X_{i}$, is omissible. Since $1=\bigcap_{j \neq i} X_{j}$, by Lemma 2.2(b), it follows from Proposition 2.4 that $|V|=9$. Now we apply Lemma 2.6. Firstly, by (c) of that result, the union (2.3) is redundant, and at most two terms on the right are omissible. If omitting $S_{5}$ say, leaves an irredundant union then, by Lemma 2.6(b), $V=X_{1} \cup X_{2} \cup X_{3} \cup X_{4}$ and $M_{5}$ is omissible from (2.2), contradiction. If omitting $S_{4}$ and $S_{5}$ from (2.3) leaves $V=S_{1} \cup S_{2} \cup S_{3}$ then Lemma 2.6(a) yields $X_{1}=X_{2}=X_{3} \subseteq D \cap V=1$, another contradiction.

Finally we note the following well known fact which is used repeatedly, and without explicit reference, throughout what follows: if $M$ is a maximal subgroup, and $U$ an Abelian minimal normal subgroup, of a group then either $U \subseteq M$ or $U \cap M=1$.

\section{Proof of TheOREM 1.1}

We have already determined the 2-groups which have maximal irredundant corefree intersection 5-covers. The next lemma addresses non-2-groups

LEMma 3.1. Suppose that the intersection of a maximal irredundant cover of five subgroups of a group $G$ is core-free. If $G$ is not a 2-group then every minimal normal subgroup of $G$ has order 4.

Proof: By Lemma 2.2(a) $G$ is a $\{2,3\}$-group. Since $G$ is soluble, by Burnside's Theorem, every minimal normal subgroup $U$ of $G$ is Abelian. Moreover, by Lemma $2.2(\mathrm{~d}),|U| \leqslant 4$.

If $|U|=2$ then, again by Lemma $2.2(\mathrm{~d}), U$ is contained in at most three of the subgroups $A_{i}$, say $U \nsubseteq A_{4} \cup A_{5}$. Since $U$ is central, and since $G=A_{4} U=A_{5} U$, every 3-element of $G$ is in $A_{4} \cap A_{4}$. However if $1 \neq u \in U$ and if $y$ is a 3-element, then $u y \notin A_{4} \cup A_{5}$. Hence $u y \in A_{1} \cup A_{2} \cup A_{3}$ and therefore $y \in A_{1} \cup A_{2} \cup A_{3}$. It follows that a Sylow 3-subgroup $S$ of $G$ is in $A_{1} \cup A_{2} \cup A_{3}$ and therefore, by Proposition 2.3, in one of $A_{i}(1 \leqslant i \leqslant 3)$, say in $A_{3}$. Therefore $S \subseteq A_{3} \cap A_{4} \cap A_{5}$ and so, by Lemma 
2.2(c), $S \subseteq D$. Since, therefore, every 3-element of $G$ is in $D$ so is the subgroup $T$ which they generate. Of course $T \unlhd G$ so $T=1$. But this contradicts the fact that $G$ is not a 2-group. Therefore $G$ has no normal subgroups of order 2 .

If $|U|=3$ then $U$ is contained in at most two of the subgroups $A_{i}$, say $U \nsubseteq$ $A_{3} \cup A_{4} \cup A_{5}$. It follows that $G=U A_{i}(3 \leqslant i \leqslant 5)$. An argument similar to that of the last paragraph shows that every 2-element of $C:=\mathbf{C}_{G}(U)$ is in $D$. Since the subgroup they generate is normal it is 1 , and we see that $C$ is a 3-group. Also, $\Phi(C) \subseteq \Phi(G) \subseteq D$, so $\Phi(C)=1$. That is, $C$ is elementary Abelian. By Lemma 2.5(b) $C \neq G$. That is, no minimal normal subgroup of $G$ is central. However $|G: C|=2$, and so $G$ satisfies the hypotheses of Lemma 2.7, contradiction.

Proof of Theorem 1.1: Let $G$ be a group with a maximal irredundant cover $\bigcup_{i=1}^{5} A_{i}$ with core-free intersection $D$. By Lemma 2.5 we may suppose that $G$ is not a 2-group. Suppose that $U$ is a minimal normal subgroup of $G$. It follows from Lemma 3.1 that $|U|=4$. Also, by Lemma $2.2(\mathrm{~d}), U$ is in at most one of the subgroups $A_{i}$, say $U \nsubseteq A_{2} \cup A_{3} \cup A_{4} \cup A_{5}$. A familiar argument gives that $C:=\mathrm{C}_{G}(U)$ is an elementary 2-group. Moreover $G / C$ embeds into Aut $(U) \cong \operatorname{Sym}_{3}$, and $O_{3}(G / C) \neq 1$. As $G / C$ module, $C$ has no non-trivial fixed points for the action of $O_{3}(G / C)$, using Lemma 3.1. It follows that $C$ is the first or second nilpotent residual of $G$. Therefore $C$ is complemented in $G$, using the result in [2, (5.18) p.383], say $G=C H$ where $H \cong C_{3}$ or $H \cong \mathrm{Sym}_{3}$. As $H$-module $C$ is completely reducible, and every minimal normal subgroup of $G$ is of order 4 .

If $C=U$ then $G \cong \mathrm{Alt}_{4}$ or $G \cong \mathrm{Sym}_{4}$. The first case is (b) of the theorem. The second does not arise because $\mathrm{Sym}_{4}$ has no maximal irredundant cover of five subgroups. For, $D$ is core-free, does not contain the monolith of $\mathrm{Sym}_{4}$ so, by Lemma 2.2(d), four of the five subgroups of the cover are copies of $\mathrm{Sym}_{3}$ whilst the fifth, therefore, contains all the elements of $\mathrm{Sym}_{4}$ of order 4 . However this is a contradiction because these elements generate $\mathrm{Sym}_{4}$.

If $C \neq U$ then $\mathbf{C}_{A_{i}}(U) \neq 1(2 \leqslant i \leqslant 5)$, and $C=U \times \mathbf{C}_{A_{i}}(U)$. Since $D$ is core-free, it follows from Lemma 3.1 and Lemma 2.2(d) that $1=\mathbf{C}_{A_{i}}(U) \cap \mathbf{C}_{A_{j}}(U)$ $(2 \leqslant i<j \leqslant 5)$. Then, for $i \neq j$,

$$
\left|\mathbf{C}_{A_{j}}(U)\right||U|=|C| \geqslant\left|\mathbf{C}_{A_{i}}(U) \mathbf{C}_{A_{j}}(U)\right|=\left|\mathbf{C}_{A_{i}}(U)\right|\left|\mathbf{C}_{A_{J}}(U)\right|
$$

so that $|U| \geqslant\left|\mathbf{C}_{A_{i}}(U)\right| \geqslant|U|$. It follows that each $\mathbf{C}_{A_{i}}(U)$ is minimal normal in $G$. That is, $C$ is the direct product of two minimal normal subgroups of $G$. If $H$ were isomorphic to $\mathrm{Sym}_{3}$ then $C$, as $H$-module, would contain just three proper non-zero submodules instead of the (at least) five it does contain. Hence $|H|=3$.

Now we examine the nature of this cover for $G$. Choose $a \in G$ of order 3. Then $\langle a\rangle$ is a Sylow 3-subgroup of $G$, and every 3-element of $G$ is conjugate either to $a$ or 
to $a^{2}$. Define $S_{i}:=\left\{w \in C: a^{w} \in A_{i}\right\}(1 \leqslant i \leqslant 5)$ and $N_{i}:=A_{i} \cap C(1 \leqslant i \leqslant 5) . S_{i}$ is a coset on $N_{i}$ in $C:$ it is not empty since otherwise $A_{i}$ would contain no 3-element, would therefore be equal to $C$, and some $N_{j}$ would be in two of the $A_{k}$ whence, by Lemma 2.2(d), in $D$, which is core-free. We have

$$
C=S_{1} \cup S_{2} \cup S_{3} \cup S_{4} \cup S_{5}
$$

since every $a^{w}$ is in some $A_{i}$. We may regard $C$ as a space of dimension 2 over the field $F$ of 4 elements, where $\langle a\rangle$ is the multiplicative group of $F$, and apply Lemma 2.6(b). If the union (3.1) is irredundant then $S_{i}=N_{i} c(1 \leqslant i \leqslant 5)$ for some $c \in C$. Hence $a^{c} \in A_{i}(1 \leqslant i \leqslant 5)$, so $|D|=3$ and $G$ has the structure required by (c) of the theorem. If, however, (3.1) is redundant then, by Lemma 2.6, at most one term, say $S_{5}$, is omissible and $N_{i}=N_{1}(1 \leqslant i \leqslant 4)$. This gives $N_{1}=\bigcap_{i=1}^{4} N_{i} \subseteq \bigcap_{i=1}^{4} A_{i}=D$, a contradiction to the core-freeness of $D$.

\section{Proof of Theorem 1.2}

If the result is false, let $G$ be a group with an irredundant cover $\mathcal{C}$ of five subgroups, with core-free intersection $D$, for which $|G: D|>16$. In the light of Theorem 1.1, $\mathcal{C}$ is not maximal. Suppose $\mathcal{C}$ chosen from among such 5-covers of $G$ with as many maximal subgroups as possible. Let $\mathcal{C}^{*}$ be a cover of $G$ got from $\mathcal{C}$ by replacing one of its nonmaximal subgroups by a maximal subgroup containing it. Write $D^{*}$ for the intersection of $\mathcal{C}^{*}: D^{*} \supseteq D$. $\mathcal{C}^{*}$ is redundant; for, if not, $D^{*}=D$ by Lemma $2.2(\mathrm{~b})$, and so is core-free, while $\mathcal{C}^{*}$ has more maximal subgroups than does $\mathcal{C}$. It follows that we may write $\mathcal{C}=\bigcup_{i=1}^{5} A_{i}$ where $A_{1}$ is not maximal, and if $A_{1}^{*}$ is a maximal subgroup containing it, then $\mathcal{C}^{*}=\left\{A_{1}^{*}, A_{2}, A_{3}, A_{4}, A_{5}\right\}$ is redundant as a cover for $G$.

If $G$ is an irredundant union of four of the subgroups in $\mathcal{C}^{*}$, then we may suppose that

$$
G=A_{1}^{*} \cup A_{2} \cup A_{3} \cup A_{4}
$$

since $A_{1}^{*}$ is certainly essential. If $D_{1}:=A_{1}^{*} \cap A_{2} \cap A_{3} \cap A_{4}$ then it follows from Proposition 2.4 that $\left|G: D_{1}\right| \leqslant 9$ with equality only if $A_{1}^{*} \cap A_{i}=D_{1} \quad(2 \leqslant i \leqslant 4)$. If we have equality therefore, it follows that

$$
A_{1}^{*}=A_{1} \cup D_{1} \cup\left(A_{5} \cap A_{1}^{*}\right)
$$

an irredundant union. However from (4.1) we deduce that $\left|A_{1}^{*}: D_{1}\right|=3$, and from (4.2) and Proposition 2.3 that $\left|A_{1}^{*}: D_{1}\right|=2$, a contradiction. Hence $\left|G: D_{1}\right| \leqslant 8$. Then, 
since $D_{1}=A_{2} \cap A_{3} \cap A_{4}$, we have $\left|D_{1}: D\right| \leqslant 2$ by Lemma $2.2(\mathrm{c})$, so $|G: D| \leqslant 16$, a contradiction.

Lastly, if $G$ is an irredundant union of three of the subgroups in $\mathcal{C}^{*}$, we may suppose that

$$
G=A_{1}^{*} \cup A_{2} \cup A_{3}
$$

since $A_{1}^{*}$ is surely included. Let us write $N:=A_{2} \cap A_{3}\left(=A_{1}^{*} \cap A_{2}=A_{1}^{*} \cap A_{3}\right)$. Now

$$
A_{1}^{*}=A_{1} \cup N \cup\left(A_{1}^{*} \cap A_{4}\right) \cup\left(A_{1}^{*} \cap A_{5}\right) .
$$

If the union (4.4) is irredundant then $\left|A_{1}^{*}: D\right|=\left|A_{1}^{*}: A_{1} \cap N \cap A_{4} \cap A_{5}\right| \leqslant 9$. However, by (4.3), $\left|A_{1}^{*}: A_{2} \cap A_{3}\right|=2$, so $\left|A_{1}^{*}: D\right| \neq 9$. Hence $|G: D|=\left|G: A_{1}^{*}\right|\left|A_{1}^{*}: D\right| \leqslant$ 16 , a contradiction. On the other hand if the union (4.4) is redundant then three of the subgroups on the right side are essential, and the possible intersections $I$ satisfy $|I: D| \leqslant 2$, using Lemma 2.2(c). Hence $|G: D|=\left|G: A_{1}^{*}\right|\left|A_{1}^{*}: I\right||I: D| \leqslant 2.4 .2=16$. This contradiction completes the proof of Theorem 1.2.

\section{REFERENCES}

[1] R.A. Bryce, V. Fedri and L. Serena, 'A Hughes-like property for finite groups', Proc. Edinburgh Math. Soc. (2) 38 (1995), 533-541.

[2] K. Doerk and T. Hawkes, Finite soluble groups (Walter de Gruyter, Berlin, New York, 1992).

[3] D. Greco, 'Su alcuni gruppi finiti che sono somma di cinque sottogruppi', Rend. Sem. Mat. Univ. Padova 22 (1953), 313-333.

[4] D. Greco, 'Sui gruppi che sono somma di quattro o cinque sottogruppi', Rend. Accad. Sci. Fis. Mat. Napoli (4) 23 (1956), 49-59.

[5] B.H. Neumann, 'Groups covered by finitely many cosets', Publ. Math. Debrecen 3 (1954), 227-242.

[6] G. Scorza, 'I gruppi che possono pensarsi come somma di tre loro sottogruppi', Boll. Un. Mat. Ital. 5 (1926), 216-218.

[7] M.J. Tomkinson, 'Groups covered by finitely many cosets or subgroups', Comm. Algebra 15 (1987), 845-859.

School of Mathematical Sciences The Australian National University Canberra ACT 0200

Australia
Dipartimento di Matematica viale Morgagni 67/A 50134 Firenze Italia 